\title{
Protective effects of cannabidiol on cuprizone-induced demyelination in C57BL/6 mice
}

\author{
Maryam Sajjadian, Iraj Ragerdi Kashani, ${ }^{*}$ Parichehr Pasbakhsh, ${ }^{1}$ Mahmoud Hassani, ${ }^{2}$ Ameneh Omidi, \\ Nasrin Takzare, ${ }^{1}$ Tim Clarner, ${ }^{4}$ Cordian Beyer, ${ }_{1}^{4}$ Adib Zendedel| ${ }^{4,5}$
}

\author{
'Department of Anatomy, School of Medicine, Tehran University of Medical Sciences, Tehran, Iran \\ 2Department of Molecular Medicine, School of Advanced Technologies in Medicine, Tehran University of Medical Sciences, Tehran, Iran \\ 'Department of Anatomical Sciences, Medical Sciences Faculty, Tarbiat Modares University, Tehran, Iran \\ ${ }^{4}$ Faculty of Medicine, Institute of Neuroanatomy, RWth Aachen University, Aachen, Germany \\ Department of Anatomical Sciences, Faculty of Medicine, Gilan University of Medical Sciences, Rasht, Iran \\ *Correspondence to Iraj Ragerdi Kashani (email: ragerdi@tums.ac.ir). \\ (Submitted: 10 June 2017 - Revised version received: 02 July 2017 - Accepted: 10 July 2017 - Published online: 26 September 2017)
}

\begin{abstract}
Objective Experimental and clinical studies suggest that oxidative stress plays an important role in the pathogenesis of multiple sclerosis. Multiple studies have shown that non-psychoactive cannabinoid, cannabidiol (CBD) exerts antioxidant effects, and has recently been approved for treatment of inflammation, pain, and spasticity associated with MS patients. The aim of this study is to determine the effectiveness of CBD in a multiple sclerosis mouse model, i.e. cuprizone-induced demyelination.

Methods Adult male BL/6 mice were fed with $0.2 \%$ cuprizone for 5 weeks which caused severe demyelination of the corpus callosum (CC). Animals were simultaneously treated with $5 \mathrm{mg} \cdot \mathrm{kg}^{-1} \mathrm{CBD}$ by daily intra-peritoneal injections. Using immunohistochemistry and transmission electron microscope, we evaluated the effects of CBD on demyelination, malondialdehyde levels and the activity of reduced glutathione, catalase and superoxide dismutase was evaluated by Biochemical analysis.

Results CBD ameliorate the cuprizone-induced demyelination and microglia accumulation. Biochemical analysis showed that oxidative stress induced by cuprizone was reduced by CBD.

Conclusion Our data implicate that CBD attenuates destructive effects of cuprizone in the CC by decreasing oxidative stress and microglia repletion.

Keywords Cannabidiol, demyelination, oxidative stress, microglia, cuprizone
\end{abstract}

\section{Introduction}

Multiple sclerosis (MS) is a severe demyelinating, chronic inflammatory disease characterized by multifocal damage of myelin in the central nervous system (CNS). ${ }^{1}$ A key study by Lucchinetti et al. ${ }^{2}$ described 4 different lesion subtypes: pattern 1 and 2 lesions are autoimmune mediated, while pattern 3 and 4 lesions are presumed to be caused by a primary oligodendrogliapathy. Pattern 3 lesions in particular display many pathological similarities with cuprizone-induced demyelination: (i) actively demyelinating lesions with little involvement of T-cells but microglia accumulation and (ii) hypoxia-like tissue damage with signs of metabolic stress and mitochondrial dysfunction which eventually leads to pronounced oligodendrocyte apoptosis. ${ }^{3} \mathrm{MS}$ has long been considered as T-cell-mediated autoimmune disease of the CNS characterized by microglia activation, recruitment of systemic immune-competent cells and production of cytotoxic mediators and pro-inflammatory cytokines, which lead to neuronal tissue damage (inflammatory cascade). ${ }^{4}$ Although controlled clinical studies published evidence for the effectiveness of immune-modulatory, antiinflammatory or immunosuppressive therapies, however this effect is mainly seen in patients at early stages of the disease, and the effects are limited, especially in improvement of the accumulation of constant clinical deficits. ${ }^{5,6}$ Therefore, there is a high need for new effective drugs that limit demyelination and axonal degeneration, thus complementing the currently available therapies. ${ }^{7}$ A number of studies suggest the importance of primary neurodegenerative mechanisms that are evident in chronically demyelinating plaques and are believed to be the result of oxidative stress. ${ }^{4,8}$ Oxidative stress basically reflects a condition in which the pro-oxidant-anti-oxidant balance in a cell is disturbed; cellular biomolecules undergo severe oxidative damage, ultimately impairing cell viability. ${ }^{9}$ The CNS is particularly sensitive to oxidative stress because of high oxygen consumption, relatively small amounts of conventional anti-oxidants and anti-oxidative enzymes, and large amounts of polyunsaturated lipids which are highly susceptible to oxidation. ${ }^{10}$

The discovery of the psychoactive effects of Cannabis sativa marked the beginning of a new field of research into the pharmacological and physiological role of the cannabinoids. ${ }^{11}$ Cannabis sativa contains a class of phytocannabinoids that include tetrahydrocannabinol (THC), the major psychoactive constituent, and cannabidiol (CBD), a non-psychoactive constituent. ${ }^{12} \mathrm{CBD}$ exerts multiple pharmacological actions in the CNS via no-CB1 and no-CB2 receptors. ${ }^{13}$ In addition to its beneficial effects on pain and spasticity associated with MS, CBD may influence the pathogenesis of MS by exerting anti-inflammatory, anti-oxidant and neuroprotective effects. ${ }^{12}$ It has been shown, that CBD can control microglia functions. Furthermore, it appears neuroprotective in a mouse model of Alzheimer disease ${ }^{14}$ and ameliorates immune-regulatory actions in both the Theiler's murine encephalitis virus (TMEV) ${ }^{15}$ and experimental autoimmune encephalomyelitis (EAE) models of MS. ${ }^{16}$

Previous studies demonstrate that cannabinoids have safety and efficacy in reducing the symptoms of multiple 
sclerosis in animal models ${ }^{17}$ and also in humans. ${ }^{18}$ To further investigate the myeloprotective prospective of $\mathrm{CBD}$, we used the potential of non-immune driven cuprizone intoxication. ${ }^{19}$ Demyelination in the cuprizone model is an early pathological event mainly affecting oligodendrocytes and axons without the breakdown of the blood brain barrier and concomitant leukocyte/blood monocyte infiltration. ${ }^{15}$ Histopathological features of the cuprizone-induced demyelination closely resemble type III MS lesions as defined by Lucchinetti et al. ${ }^{2}$ and oxidative stress was shown to be a key factor in cuprizone pathophysiology. ${ }^{20}$ The goal of this study was to evaluate the anti-oxidant effects of CBD during experimentally- induced demyelination by cuprizone in the corpus callosum (CC) of young male mice.

\section{Materials and Methods}

\section{Animals}

Research and animal care were approved by the Review Board for the Care of Animal Subjects of the district government (Tehran, Iran). In vivo experiments were performed with 8 weeks old male C57BL/6 mice (approx. 20 g, Pasteur, Iran) Animals were housed under temperature-controlled conditions with a 12/12-hour light-dark cycle and ad libitum access to water and food.

\section{Cuprizone-induced Demyelination and CBD Treatment}

In this study cuprizone as an oral agent was used that causes acute, fast recovering demyelinated lesions. ${ }^{21}$ Eight-week-old $(20 \mathrm{~g})$ male $\mathrm{C} 57 \mathrm{Bl} / 6$ mice were randomly divided into four groups ( $n=10$ per group). One group was fed normal diet and served as controls. The three other groups received diets containing $0.2 \%(\mathrm{w} / \mathrm{w})$ cuprizone (Sigma, USA) to induce demyelination. CBD (Sigma, USA) was dissolved in PBS which served as vehicle. The vehicle plus CBD complex (5 $\left.\mathrm{mg} \cdot \mathrm{kg}^{-1}\right)$ was injected intraperitoneally into cuprizone-treated mice (CPZ+C group) every other day for 5 weeks. The chosen CBD dosage closely resembled that used in another study to treat $\mathrm{CPZ}$ model of MS in mice. ${ }^{22}$ The 4 groups were as follows: control group that was fed normal diet, cuprizone group which was given only $\mathrm{CPZ}$, vehicle-treated cuprizone group $(\mathrm{CPZ}+\mathrm{V})$ and cannabidiol-treated cuprizone group $(\mathrm{CPZ}+\mathrm{C})$. Mice were anaesthetized with ketamine $(115 \mathrm{mg} / \mathrm{kg})$ (Sigma, USA) and xylazine $(10 \mathrm{mg} / \mathrm{kg}$ ) (Sigma, USA) and killed after 5 weeks. Animal handling and treatment protocols have been previously described in detail. ${ }^{4}$ For IHC and biochemical studies, the numbers of animals in the experimental groups were as follows: control $(n=4), \mathrm{CPZ}(n=5), \mathrm{CPZ}$ plus $\mathrm{CBD}(n=6)$.

\section{Luxol Fast Blue Staining}

Myelination was analyzed in sections by luxol fast blue staining (LFB, Sigma, USA). Sections were placed overnight in LFB at $56^{\circ} \mathrm{C}$ and washed in $95 \%$ ethanol and distilled water to remove excess blue stain. The color was then differentiated (until white matter was easily distinguishable from gray matter) in a lithium carbonate solution for $15 \mathrm{~s}$, followed by distilled water and three $80 \%$ alcohol washing steps. Slices were further passed through fresh xylene twice, mounted with Entellan (Merck, Germany). In order to evaluate sections based upon demyelination, LFB stained sections were scored between zero and three, by three independent, blinded readers. A score of three is equivalent to a normal myelin status, whereas zero is referred to maximum demyelinated CC. A score of one or two corresponds to one-third or two-third fiber myelination of the CC, respectively.

\section{Transmission Electron Microscopy}

For electron microscopic examination, three animals per group were transcardially perfused with $2 \%$ glutaraldehyde and $2 \%$ paraformaldehyde in $0.1 \mathrm{M}$ PBS. Brains were quickly removed and placed on ice. The CC was dissected, and the samples were placed in a tube. Samples were then transferred immediately after extraction in $2.5 \%$ glutaraldehyde (Fluka) in $0.1 \mathrm{M}$ cacodylate buffer $(\mathrm{pH} 7.4)$ at $4^{\circ} \mathrm{C}$ overnight and transferred to $1 \%$ osmium tetroxide in the same buffer for $1 \mathrm{~h}$ at room temperature. Tissue was transversely cut into $1 \mathrm{~mm}$ blocks which were further fixed in osmium tetroxide at $4^{\circ} \mathrm{C}$ overnight, dehydrated through ascending ethanol washes, and embedded in epoxy resin (TAAB laboratories). $1 \mu \mathrm{m}$ sections were cut, stained with toluidine blue, and examined by light microscopy to identify demyelinated areas. Selected areas were subsequently examined by TEM (IEO 906 Germany, $100 \mathrm{kV})$.

According to the method of Zambonin and et al..$^{23}$, sagittal sections of the CC were submitted to a quantitative evaluation to determine the percentage of correctly myelinated axons per field. CC images were taken from 4 sections with an intersectional distance of $50 \mu \mathrm{m}$. Photographs of axons orientated in cross-section were taken, and quantification of myelinated axons was performed on 4 images per animal and treatment at a magnification of $x 3500$ using the Image J software.

\section{Immunohistochemistry}

After anesthesia, the animals were transcardially perfused with PBS followed by $4 \%$ paraformaldehyde (PFA, Sigma, Germany) in 0.1 M PBS, pH 7.4. Brains were removed, postfixed in 4\% PFA overnight and rinsed with PBS. After overnight post-fixation, brains were processed, embedded, and sectioned into $5 \mu \mathrm{m}$ sections from the levels $215-275$ as stated in the mouse brain atlas by Sidman et al. (http:// www.hms.harvard.edu/research/brain/atlas.html). For IHC, sections were placed on silane-coated slides, de-paraffinized, rehydrated, heat-unmasked and blocked with PBS containing $5 \%$ normal serum. Afterwards, sections were exposed to antiionized calcium binding adaptor molecule (anti-Iba-1, 1:4.000; Wako, Germany) for overnight at $4^{\circ} \mathrm{C}$. The next day, sections were treated with $\mathrm{H}_{2} \mathrm{O}_{2} / \mathrm{PBS}$ ( $0.3 \%$, Roth, Germany) to block the endogenous peroxidase. Then, sections were incubated with the appropriate secondary antibodies followed by the Avidin-Biotin Complex (ABC) Method. Diaminobenzidine (DAB) was used as chromogen. Finally, sections were dehydrated in graded alcohols and mounted.

\section{Biochemical Analysis}

In order to recognize the effects of free radical-mediated following cuprizone-induced demyelization with or without CBD treatment, determination of reduced glutathione (GSH), lipid peroxidation (LPO), catalase (CAT) and superoxide dismutase (SOD) tissue levels were carried out in the CC. To this end, mice were transcardially perfused with 0.1 M PBS (PBS, Sigma, USA). Following dissection of the brains, the CCs were 
isolated. After that, tissue samples were homogenized on ice by application of a tissue homogenizer (Remi, India). LPO products (malondialdehyde; MDA) in the $\mathrm{CC}$ were measured according to Kashani et al. ${ }^{22}$ Briefly, tissues homogenates were produce in $0.15 \mathrm{M} \mathrm{KCl}(5 \% \mathrm{w} / \mathrm{v}$ homogenate). Microcentrifuge tubes each containing $0.6 \mathrm{ml}$ were incubated $1 \mathrm{~h}$ at $37^{\circ} \mathrm{C}$. Following that, $1.2 \mathrm{ml}$ of $28 \% \mathrm{w} / \mathrm{v}$ trichloroacetic acid (TCA) solution (5\%) was added, and by adding $1.2 \mathrm{ml}$ of water, the final volume was reached to $3 \mathrm{ml}$. Subsequently, centrifugation at $3000 \mathrm{xg}$ for $10 \mathrm{~min}$ was performed and $2.5 \mathrm{ml}$ of the supernatant was collected. After that, the color was developed by addition of $0.5 \mathrm{~mL}$ of $1 \% \mathrm{w} / \mathrm{v}$ thiobarbituric acid dissolved in $0.05 \mathrm{~N} \mathrm{NaOH}$ keeping the solution in boiling water bath for 15 min until the appearance of pink color. Finally, the absorbance was read in a spectrophotometer at $532 \mathrm{~nm}$. MDA contents were declared as nmol/g wet tissue. The GSH content of CC was also determined by spectrophotometer. Briefly, proteins from homogenized tissues (10\% w/v in PBS, $\mathrm{pH} 7.4)$ were removed, and after adding an equal volume of $10 \%$ TCA, incubated at $4^{\circ} \mathrm{C}$ for $2 \mathrm{~h}$. Then, the samples were centrifuged at $2000 \mathrm{xg}$ for $15 \mathrm{~min}$, and the supernatant was added to $2 \mathrm{ml}$ of 0.4 M Tris buffer ( $\mathrm{pH}$ 8.9) including 0.02 M EDTA ( $\mathrm{pH} 8.9$ ) (Sigma, USA). After that, 0.01 M 5,5'-Dithio-bis 2-nitrobenzoic acid was added. Eventually, the mixture was diluted with 0.5 $\mathrm{ml} \mathrm{DW}$, and absorbance was read in a spectrophotometer at $412 \mathrm{~nm}$. Results are shown as $\mu \mathrm{g}$ GSH/g wet tissue sample. Total SOD activity in the CC homogenate was measured based on the ability of SOD to inhibit the production of formazan dye resulting from the reaction of WST-1 (water-soluble tetrazolium salt) and superoxide anion. Briefly, samples were mixed with WST-1 solution and enzyme solution (xanthine oxidase) and incubated at $37^{\circ} \mathrm{C}$ for $30 \mathrm{~min}$. Then the absorbance of the solution at $450 \mathrm{~nm}$ was measured. The activity of total SOD in the brain tissue was calculated by referring to the standard curve and expressed as $\mathrm{U} / \mathrm{mg}$ protein.

\section{Data analysis}

All data are given as means \pm SD. Statistical differences between various groups were analyzed by one-way analysis of variance (ANOVA) followed by Tukey's post hoc test using GraphPad Prism 7 (GraphPad Software Inc., USA).

\section{Results}

\section{CBD treatment delays demyelination}

Standard LFB staining revealed a normal myelin structure in the control group (Fig. 1A). The animals treated with cuprizone and PBS (CPZ+V group) or without PBS (CPZ) exhibited a significant decrease in LFB staining, characterized by bright areas indicative of myelin disorganization (Fig. 1B, C and E). In the animals treated with cuprizone plus $\mathrm{CBD}(\mathrm{CPZ}+\mathrm{C}$ group), LFB staining was significantly stronger than in the $\mathrm{CPZ}$ group but the myelin aspect was not uniform and displayed vacuoles (Fig. $1 \mathrm{D}$ and $\mathrm{E}, P \leq 0.05$ ).

\section{Cannabidiol effects on the myelination index}

TEM photographs were used to determine the percentage of myelinated axons in the CC. Under control conditions, we found nearly the whole axon population as densely packed axons with a typical range in diameter and homogeneous regular myelin sheets (Fig. 2A). Cuprizone treatment with or

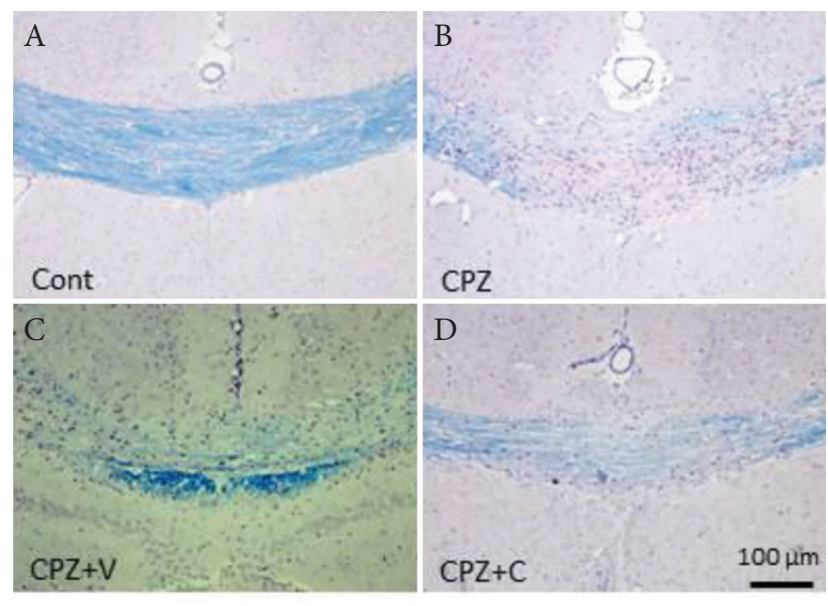

E

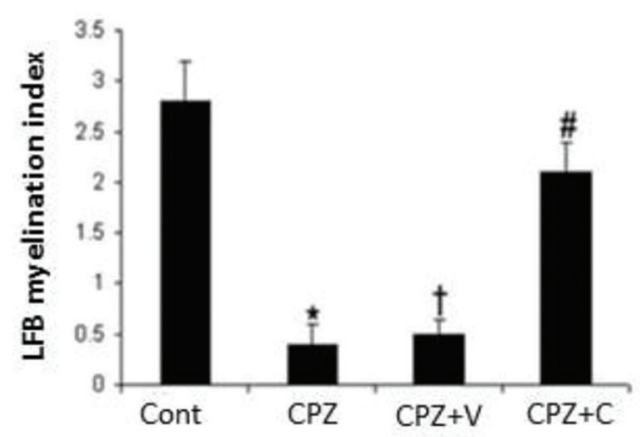

Fig. 1 Demyelination in the corpus callosum (CC) after 5 weeks of cuprizone-induced demyelination with or without cannabidiol treatment. Myelination index was evaluated in LFB- stained sections. Photomicrographs were taken from coronal sections of the CC. (A) control, (B) shows cuprizone-induced demyelination, (C) cuprizone with vehicle, (D) cuprizone-induced demyelination in the cannabidiol-treated group. Quantification of the myelination index in the center of the CC reveals a significant myelin loss in the cuprizone group and a restoration of myelin in the group co-treated with cannabidiol. Data represented means $\pm S D$. ${ }^{*} P \leq 0.05 C P Z$ vs. control, ${ }^{\dagger} P \leq 0.05$ CPZ+V vs. control, ${ }^{\#} P \leq 0.05$ CPZ+C vs. CPZ+V. Scale bar $100 \mu \mathrm{m}$.

without PBS resulted in a massive reduction in myelinated axons numbers up to $90 \%$ (Fig. 2 B and C). We also observed very small myelinated axons with frazzled myelin layers. The co-administration of CBD partially inhibited the mentioned decline (Fig. $2 \mathrm{D}$ and $\mathrm{E}, P \leq 0.05$ cuprizone versus cuprizone and $\mathrm{CBD})$.

\section{CBD effect on microglia accumulation}

Microglia accumulation was evaluated by IHC against Iba-1. Quantification of Iba-1 staining intensity in the CC showed that in control animals, few microglia and low staining intensity can be detected (Fig. 3A and E). In contrast, the area of Iba-1 staining and its intensity significantly increased in the $\mathrm{CPZ}$ and $\mathrm{CPZ}$ plus vehicle animals (Fig. 3B-E, $P \leq 0.05$ ). Staining values for Iba-1 were significantly lower in $\mathrm{CPZ}$ plus CBD animals (Fig. 3D and $\mathrm{E}, P \leq 0.05$ ).

\section{MDA and levels of anti-oxidants}

In order to analyze the effects of cuprizone and CBD exposure on parameters distinctive for oxidative stress, we determined of MDA, GSH, CAT and SOD levels in homogenates of the CC. As shown in Fig. 4, the activities of antioxidant enzymes (GSH, CAT and SOD) decreased significantly (after 

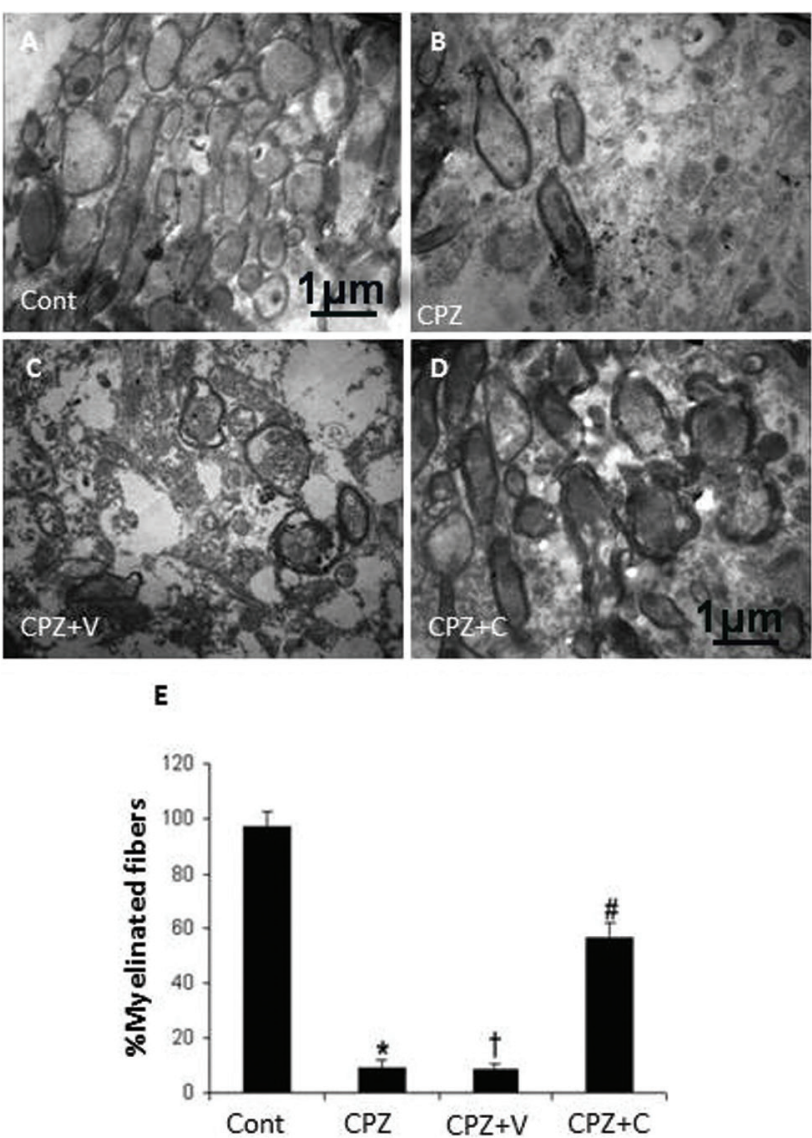

Fig. 2 Representative TEM micrographs of axons in the corpus callosum (CC). For details of abbreviations of the groups see legend of Figure 1. Micrographs show a massive decrease in the number of axons in the CC after cuprizone and a partial restoration of axon numbers and myelination after cannabidol. Data represent means $\pm S D,{ }^{*} P \leq 0.05$. Scale bar $1 \mu \mathrm{m}$.

cuprizone-induced demyelination without CBD treatment compared with the control group (Fig. 4B-D). The level of the anti-oxidant enzymes (GSH, CAT and SOD) was significantly increased $(P \leq 0.05)$ after cuprizone-induced demyelination with $\mathrm{CBD}$ treatment $(\mathrm{CPZ}+\mathrm{C}$ group) compared with the cuprizone-induced demyelination with $\mathrm{PBS}$ as a vehicle ( $\mathrm{CPZ}+\mathrm{V}$ group). In contrast, MDA levels were significantly increased after cuprizone-induced demyelination without CBD treatment $(22.1 \pm 2.9)$ compared with control mice (Fig. $4 \mathrm{~A})$. Interestingly, cuprizone-induced demyelination with $\mathrm{CBD}$ treatment $(\mathrm{CPZ}+\mathrm{C}$ group) significantly $(P \leq 0.05)$ decreased MDA levels compared with cuprizone-induced demyelination without CBD (Fig. 4A).

\section{Discussion}

In the present study, we demonstrated that the intraperitoneal administration of CBD significantly ameliorate demyelination in the CC, decreased microglia accumulation and alleviated oxidative stress after cuprizone-intoxication.

Recent years have provided evidence that oxidative stress plays an important role in the pathogenesis of MS. ${ }^{1,2,5}$ Here, we used the cuprizone mouse model which is known to cause oxidative damage of oligodendrocytes and dystrophic axons in the brain which closely mimics to some extent the so-called pattern III lesions in MS patients. ${ }^{22,24}$ Our results show that

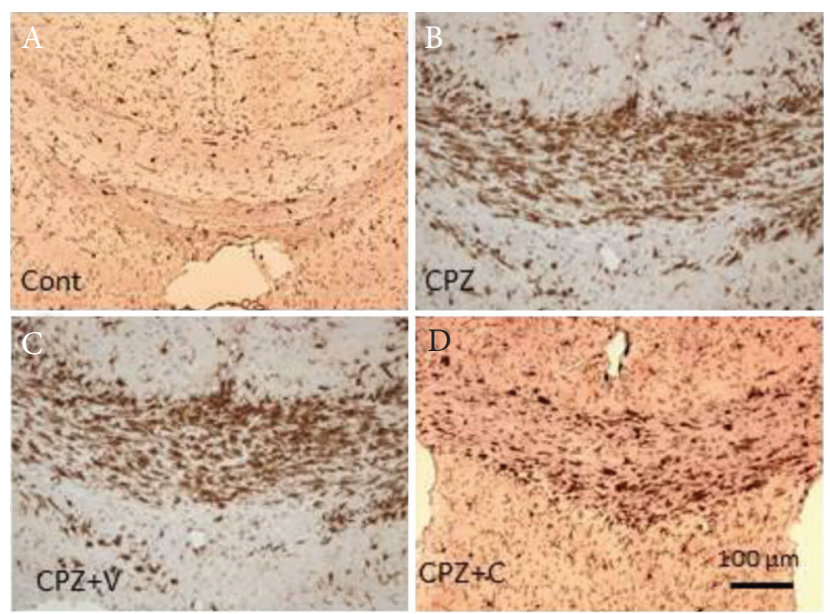

$\mathrm{E}$

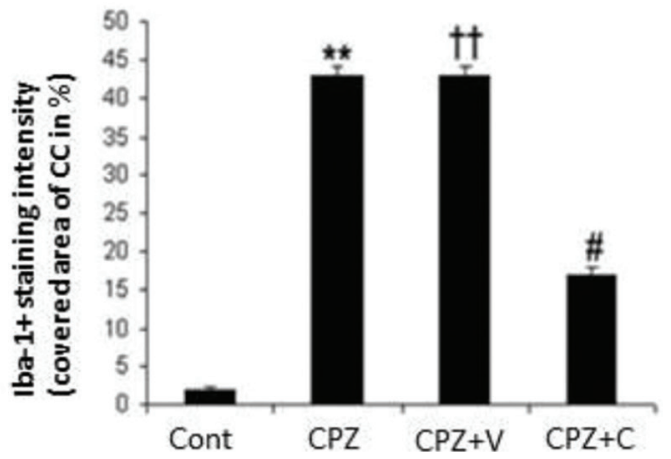

Fig. 3 Effect of cuprizone and cannabidiol treatment on microglial accumulation in the midline of the corpus callosum (CC). For details of abbreviations of the groups see legend of Fig. 1. IHC revealed that in control animals, only few lba-1+ cells can be found $(A, E)$, whereas cuprizone-induced demyelination without cannabidiol treatment (CPZ) significantly increased the number of lba-1-positive staining intensity (B, C, and E). Cannabidiol treatment significantly attenuated the cuprizone-induced microglial accumulation $(D, E)$. Data represented means $\pm S D .{ }^{* *} P \leq 0.001$ CPZ vs. control, ${ }^{+\dagger} P \leq 0.05 C P Z+V$ vs. control, ${ }^{\#} P \leq 0.05 C P Z+C$ vs. CPZ+V. Scale bar, $100 \mu \mathrm{m}$.
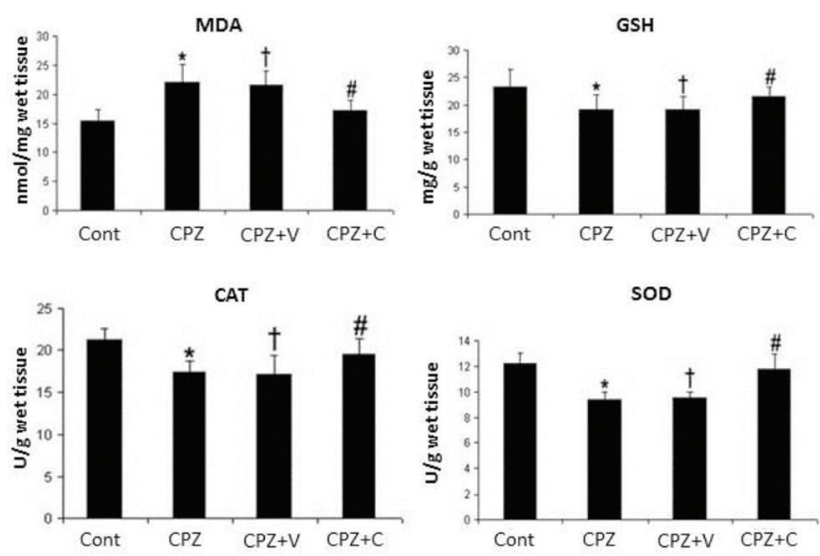

Fig. 4 Effect of cuprizone and cannabidiol on the levels of malondialdehyde (MDA), reduced glutathione (GSH), catalase (CAT) and superoxide dismutase (SOD) in the corpus callosum (CC). Cuprizone reduced GSH, CAT and SOD but increased MDA compared with control. The application of cannabidiol reversed the effect on GSH, CAT and SOD. Cannabidiol exposure itself lowered MDA levels compared with the control group. Data represented mean \pm SD. ${ }^{*} P \leq 0.05$ CPZ vs. control, ${ }^{\dagger} P \leq 0.05 C P Z+V$ vs. control, ${ }^{*} P \leq 0.05$ $\mathrm{CPZ}+\mathrm{C}$ vs. $\mathrm{CPZ}+\mathrm{V}$. 
cuprizone feeding significantly increased MDA and decreased the activities of a set of anti-oxidant enzymes/factors such as SOD, GSH and CAT. These results are in good agreement with observations from other authors. ${ }^{15,22,24-26}$ Cuprizone is able to increase levels of MDA, a routine index of lipid peroxidation, and simultaneously decrease GSH levels and antioxidant enzyme activity (CAT, SOD). ${ }^{3}$ Although the underlying mechanisms of ROS-dependent myelin loss are not yet clear, there are several plausible explanations. First, ROS can directly induce the apoptosis of oligodendrocytes, thereby leading to demyelination in $\mathrm{MS}^{23}$ Second, oligodendrocyte precursor cells may be more sensitive to oxidative stress compared to mature oligodendrocytes and thus limit CNS repair and remyelination. ${ }^{28}$ Third, ROS has direct effects on the lipid and protein components of myelin through peroxidation, and degrades myelin basic protein $(\mathrm{MBP})$ through the production of matrix metalloproteinases. ${ }^{27,28}$

We demonstrate that CBD partially reverses these effects. It is assumed that $\mathrm{CBD}$ is able to restore the normal balance between oxidative stress markers and anti-oxidant endogenous mechanisms that is often disrupted in neurodegenerative disorders. ${ }^{29}$ Anti-oxidant effects of CBD may involve intracellular mechanisms that enhance the ability of endogenous anti-oxidant enzymes to control oxidative stress, in particular the signaling triggered by the transcription factor Nuclear Factor Erythroid 2-related Factor 2 (Nrf2). ${ }^{29,30}$ Nrf2 (also known as NFE2L2) is a critical regulator of genes involved in the detoxification of reactive oxygen species. ${ }^{30} \mathrm{CBD}$ is thought to bind to an intracellular target which plays a major role in the control of anti-oxidant-response elements located in genes encoding for different anti-oxidant enzymes of the so-called phase II-anti-oxidant response and is involved in the regulation of Nrf2. ${ }^{29}$ Cuprizone treatment causes a significantly increase in the number of Iba-1 positive cells in the CC, thus being indicative for local neuroinflammation. ${ }^{4}$ Another relevant finding of our study was that CBD administration attenuated the microglia accumulation in the CC of cuprizone-treated mice. This result is consistent with other data which shown that $\mathrm{CBD}$ administration reduced microglia accumulation in different brain injury models. ${ }^{31-34}$ It seems safe to conclude that microglia plays a detrimental role in the demyelinated lesion. ${ }^{32,34}$ Microglia are the resident immune cells of the CNS, participating in the regulation of immune responses due to their ability to present antigens and to secrete immuneregulatory factors such as neurotrophins, chemokines, cytokines, and ROS. ${ }^{31,34}$ Microglia can recruit and reactivate T cells in the CNS and release detrimental molecules such as free radicals, inflammatory cytokines, and proteases, which then contribute to demyelination through a variety of different pathogenic processes. ${ }^{33} \mathrm{Wu}$ et al. showed that the production of ROS by microglia occurred primarily by NOX activation and required the Hv1 proton channel. ${ }^{35}$ Ablation of microglia or impairment of their function decreased disease progression in experimental MS animal models. ${ }^{34,36}$ Thus, suppression of microglia will potentially reduce inflammatory lesions and limit demyelination within the CNS. In addition, Mecha et al. found that CBD induced apoptosis of microglial cells through lipid raft involvement. ${ }^{31,37}$ Lipid rafts are regions of plasma membranes with a distinct, characteristic structural composition, that participate in various cellular functions, including protein trafficking, transcytosis, endocytosis, cell survival, and cell death. ${ }^{37,38} \mathrm{CBD}$ induces lipid raft coalescence, which has been linked to critical signaling pathways, such as apoptosis. $^{37-39}$

\section{Conclusion}

Our data implicate that $\mathrm{CBD}$ can attenuate destructive cuprizone effects in the brain by decreasing oxidative stress and reducing microglia accumulation. It remains to be analyzed in further studies whether such protective effects are also operant in human MS patients and are suited as therapeutic options.

\section{Acknowledgments}

The study was supported by a grant from the Tehran University of Medical sciences and health services, Tehran, Iran (Grant No. 21616-30-02-92).

\section{Conflict of Interest}

There is no conflict of interest.

\section{References}

1. Suneetha A. Role of dimethyl fumarate in oxidative stress of multiple sclerosis: a review. Journal of Chromatography B. 2016;1019:15-20.

2. Lucchinetti C, Bruck W, Parisi J, Scheithauer B, Rodriguez M, Lassman $H$. Heterogeneity of multiple sclerosis lesions: implications for the pathogenesis of demyelination. Annals of neurology. 2000;47(6):707-17.

3. Praet J, Guglielmetti C, Berneman Z, Van der Linden A, Ponsaerts P. Cellular and molecular neuropathology of the cuprizone mouse model: clinical relevance for multiple sclerosis. Neuroscience \& Biobehavioral Reviews. 2014;47:485-505.

4. Zendedel A, Kashani IR, Azimzadeh M, Pasbakhsh P, Omidi N, Golestani $A$, et al. Regulatory effect of triiodothyronine on brain myelination and astrogliosis after cuprizone-induced demyelination in mice. Metabolic brain disease. 2016:31(2):425-33.

5. Lassmann $\mathrm{H}$. What drives disease in multiple sclerosis: inflammation or neurodegeneration? Clinical and Experimental Neuroimmunology. 2010;1(1):2-11.

6. Wiendl H, Hohlfeld R. Multiple sclerosis therapeutics Unexpected outcomes clouding undisputed successes. Neurology. 2009;72(11):1008-15.

7. Janssens K, Maheshwari A, Van den Haute C, Baekelandt V, Stinissen P, Hendriks JJ, et al. Oncostatin M protects against demyelination by inducing a protective microglial phenotype. Glia. 2015;63(10):1729-37.

8. Mahad DH, Trapp BD, Lassmann H. Pathological mechanisms in progressive multiple sclerosis. The Lancet Neurology. 2015;14(2):183-93.

9. Sinha K, Das J, Pal PB, Sil PC. Oxidative stress: the mitochondria-dependent and mitochondria-independent pathways of apoptosis. Archives of toxicology. 2013;87(7):1157-80

10. Carvalho AN, Lim JL, Nijland PG, Witte ME, Van Horssen J. Glutathione in multiple sclerosis: more than just an antioxidant? Multiple Sclerosis Journal. 2014;20(11):1425-31.

11. Gomez O, Sanchez-Rodriguez A, Le M, Sanchez-Caro C, Molina-Holgado F, Molina-Holgado E. Cannabinoid receptor agonists modulate oligodendrocyte differentiation by activating PI3K/Akt and the mammalian target of rapamycin (mTOR) pathways. British journal of pharmacology. 2011:163(7):1520-32

12. Cabral GA, Jamerson M. Marijuana use and brain immune mechanisms. Int Rev Neurobiol. 2014;118:199-230.

13. Giacoppo S, Galuppo M, Pollastro F, Grassi G, Bramanti P, Mazzon E. A new formulation of cannabidiol in cream shows therapeutic effects in a mouse model of experimental autoimmune encephalomyelitis. DARU Journal of Pharmaceutical Sciences. 2015;23(1):48.

14. Martín-Moreno AM, Reigada D, Ramírez BG, Mechoulam R, Innamorato N Cuadrado A, et al. Cannabidiol and other cannabinoids reduce microglial 
activation in vitro and in vivo: relevance to Alzheimer's disease. Molecular pharmacology. 2011;79(6):964-73.

15. Acs P, Kipp M, Norkute A, Johann S, Clarner T, Braun A, et al. 17ß-estradiol and progesterone prevent cuprizone provoked demyelination of corpus callosum in male mice. Glia. 2009;57(8):807-14.

16. Kozela E, Lev N, Kaushansky N, Eilam R, Rimmerman N, Levy R, et al. Cannabidiol inhibits pathogenic $T$ cells, decreases spinal microglial activation and ameliorates multiple sclerosis-like disease in C57BL/6 mice. British journal of pharmacology. 2011;163(7):1507-19.

17. Baker D, Pryce G, Croxford JL, Brown P. Cannabinoids control spasticity and tremor in a multiple sclerosis model. Nature. 2000;404(6773):84.

18. Wade DT, Makela P, Robson P, House H, Bateman C. Do cannabis-based medicinal extracts have general or specific effects on symptoms in multiple sclerosis? A double-blind, randomized, placebo-controlled study on 160 patients. Multiple Sclerosis Journal. 2004;10(4):434-41.

19. Gudi V, Gingele S, Skripuletz T, Stangel M. Glial response during cuprizoneinduced de-and remyelination in the CNS: lessons learned. Frontiers in cellular neuroscience. 2014;8:73.

20. Draheim T, Liessem A, Scheld M, Wilms F, Weißflog M, Denecke B, et al. Activation of the astrocytic Nrf2/ARE system ameliorates the formation of demyelinating lesions in a multiple sclerosis animal model. Glia. 2016

21. Zendedel A, Beyer C, Kipp M. Cuprizone-induced demyelination as a tool to study remyelination and axonal protection. Journal of molecular neuroscience. 2013;51(2):567-72.

22. Kashani IR, Rajabi Z, Akbari M, Hassanzadeh G, Mohseni A, Eramsadati MK, et al. Protective effects of melatonin against mitochondrial injury in a mouse model of multiple sclerosis. Experimental brain research. 2014;232(9):2835-46.

23. Zambonin JL, Zhao C, Ohno N, Campbell GR, Engeham S, Ziabreva I, et al. Increased mitochondrial content in remyelinated axons: implications for multiple sclerosis. Brain. 2011;134(7):1901-13.

24. Ljubisavljevic S. Oxidative stress and neurobiology of demyelination. Molecular neurobiology. 2016;53(1):744-58

25. Gilgun-Sherki Y, Melamed E, Offen D. The role of oxidative stress in the pathogenesis of multiple sclerosis: the need for effective antioxidant therapy. Journal of neurology. 2004;251(3):261-8.

26. Miljkovi' D, Bla'evski J, Petkovi'F, Djedovi' N, Mom'ilovi' M, Stanisavljevi' S, Jevti'B, Stojkovi' MM, Spasojevi'I. A Comparative Analysis of Multiple Sclerosis-Relevant Anti-Inflammatory Properties of Ethyl Pyruvate and Dimethyl Fumarate. The Journal of Immunology. 2015;194(6):2493-503.

27. Witherick J, Wilkins A, Scolding N, Kemp K. Mechanisms of oxidative damage in multiple sclerosis and a cell therapy approach to treatment. Autoimmune diseases. 2010;2011.
28. Liu J, Tian D, Murugan M, Eyo UB, Dreyfus CF, Wang W, et al. Microglial Hv1 proton channel promotes cuprizone-induced demyelination through oxidative damage. Journal of neurochemistry. 2015;135(2):347-56.

29. Fernández-Ruiz J, Sagredo O, Pazos MR, García C, Pertwee R, Mechoulam $R$, et al. Cannabidiol for neurodegenerative disorders: important new clinical applications for this phytocannabinoid? British journal of clinical pharmacology. 2013;75(2):323-33.

30. Schneider K, Valdez J, Nguyen J, Vawter M, Galke B, Kurtz TW, et al. Increased Energy Expenditure, Ucp1 Expression, and Resistance to Diet-induced Obesity in Mice Lacking Nuclear Factor-Erythroid-2-related Transcription Factor-2 (Nrf2). Journal of Biological Chemistry. 2016;291(14):7754-66.

31. Toth CC, Jedrzejewski NM, Ellis CL, Frey WH. Cannabinoid-mediated modulation of neuropathic pain and microglial accumulation in a model of murine type I diabetic peripheral neuropathic pain. Molecular pain. 2010;6(1):1.

32. Gomes FV, Llorente R, Del Bel EA, Viveros M-P, López-Gallardo M, Guimarães FS. Decreased glial reactivity could be involved in the antipsychotic-like effect of cannabidiol. Schizophrenia research. 2015;164(1):155-63.

33. Rawji KS, Yong VW. The benefits and detriments of macrophages/microglia in models of multiple sclerosis. Clinical and Developmental Immunology. 2013;2013.

34. Heppner FL, Greter M, Marino D, Falsig J, Raivich G, Hövelmeyer N, et al. Experimental autoimmune encephalomyelitis repressed by microglial paralysis. Nature medicine. 2005;11(2):146-52.

35. Wu LJ. Microglial voltage-gated proton channel $\mathrm{Hv} 1$ in ischemic stroke. Translational stroke research. 2014;5(1):99-108.

36. Huitinga I, Ruuls S, Jung S, Rooijen Nv, Hartung H, Dijkstra C. Macrophages in $T$ cell line-mediated, demyelinating, and chronic relapsing experimental autoimmune encephalomyelitis in Lewis rats. Clinical \& Experimental Immunology. 1995;100(2):344-51.

37. Chen YC, Kung FL, Tsai IL, Chou TH, Chen IS, Guh JH. Cryptocaryone, a natural dihydrochalcone, induces apoptosis in human androgen independent prostate cancer cells by death receptor clustering in lipid raft and nonraft compartments. The Journal of urology. 2010;183(6):2409-18.

38. Wu HY, Goble K, Mecha M, Wang CC, Huang CH, Guaza C, et al. Cannabidiolinduced apoptosis in murine microglial cells through lipid raft. Glia. 2012;60(7):1182-90

39. Mecha M, Feliú A, Carrillo-Salinas F, Rueda-Zubiaurre A, OrtegaGutiérrez S, de Sola RG, et al. Endocannabinoids drive the acquisition of an alternative phenotype in microglia. Brain, behavior, and immunity. 2015;49:233-45.

This work is licensed under a Creative Commons Attribution-NonCommercial 3.0 Unported License which allows users to read, copy, distribute and make derivative works for non-commercial purposes from the material, as long as the author of the original work is cited properly. 